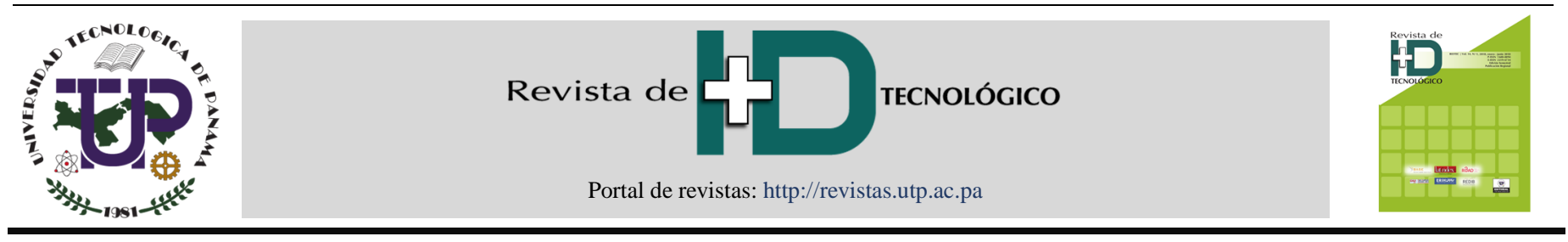

\title{
Estudio del período de vibración de puentes con vigas de acero en Panamá
} Study of vibration period for bridges with steel beams in Panama

\author{
Álvaro E. García 1* Fátima H. Garrido ${ }^{2}$, José M. Gallardo ${ }^{3}$ \\ ${ }^{1,2,3}$ Grupo de investigación: Salud Estructural de Puentes, ${ }^{1,2,3}$ Universidad Tecnológica de Panamá, Panamá \\ *Autor de correspondencia: jose.gallardo@utp.ac.pa
}

RESUMEN-En este artículo se presenta la frecuencia de vibración principal de 31 luces de puentes de viga de acero, y se estudian en conjunto con la frecuencia de vibración de otras 25 luces de puentes de acero presentadas en estudios anteriores. Se presenta una ecuación que estima el período de vibración principal en función de la longitud de la luz estudiada. En algunos puentes se observó una sincronización de la frecuencia de vibración en tramos adyacentes (los cuales presentan diferentes longitudes entre ellos) en puentes de varias luces con vigas no continuas; se propone que este fenómeno está relacionado con la vibración forzada en los tramos más cortos, causada por la vibración natural de las luces adyacentes más largas. Además, una ecuación para estimar el período de vibración que se puede utilizar como límite superior, una referencia para identificar puentes atípicos, cuyos períodos de vibración son considerablemente mayores a la tendencia.

Palabras clave- Aceleración, acero, frecuencia, periodo, puentes, vibración.

ABSTRACT - In this article the main frequency of vibration obtained in 31 spans of steel beam bridges are presented, and studied together with other 25 steel beam bridges vibration frequencies presented in previous studies. An equation that estimates the main period of vibration as a function of the span length of these bridges is presented. Additionally, matching of frequency of vibration in adjacent spans with different lengths was observed in some non-continuous-beam multi-span bridges; these phenomena is considered to be related to forced vibration in the shorter spans caused by the natural vibration of the longest spans. In addition, an equation to estimate an upper bound period of vibration as a reference to identify atypical bridges, whose vibration periods are considerably far from the trend.

Keywords-Acceleration, steel, frequency, period, bridges, vibration.

\section{Introducción}

Los puentes están sometidos constantemente a cargas dinámicas en pequeños lapsos de tiempo que provocan en ellos efectos vibratorios. La vibración libre de una estructura depende de su masa y su rigidez, la frecuencia aumenta con el aumento de la rigidez, la frecuencia disminuye con el aumento de la masa. La masa de un puente depende de la geometría de la estructura y la densidad de los materiales que la conforman, y la rigidez depende de la geometría y la relación esfuerzodeformación de los materiales que componen la estructura.

En general, la rigidez de los puentes varía según su longitud (el desplazamiento aumenta cuando la luz libre aumenta), y es principalmente dependiente de elementos estructurales longitudinales como vigas, cerchas, arcos, y los elementos que actúen en acción compuesta con estos elementos (e.g. losa, barreras vehiculares de concreto). La masa movilizada durante la vibración del puente, bajo la excitación del paso vehicular, es, principalmente la masa de los tableros, las vigas, arcos y cerchas, y las barreras vehiculares.

Cuando los elementos estructurales sufren daños que causan reducción en su rigidez (e.g. fisuras, pandeo), la estructura puede presentar cambios en su frecuencia o modo de vibración. En estudios anteriores se ha determinado que el modo de vibración de una estructura puede cambiar cuando su integridad física se encuentra comprometida $[1,2]$.

Las pruebas de carga dinámicas no destructivas facilitan la evaluación de la respuesta de un puente provocada por excitaciones predeterminadas sin cambiar la respuesta elástica de la estructura. Esta técnica ayuda a revisar el desempeño y el rendimiento del puente y sus componentes $[3,4]$.

El análisis espectral de una señal tiene como objetivo examinar, de forma minuciosa, el comportamiento y la participación de sus componentes armónicas en el dominio de la frecuencia [5].

Citación: A.García, F.Garrido y J. Gallardo "Estudio del período de vibración de puentes con vigas de acero en Panamá" Revista de I+D Tecnológico, vol. 16, no. 1, pp. (12-21), 2020.

Tipo de artículo: Original. Recibido: 15 febrero de 2019. Recibido con correcciones: 15 febrero de 2019. Aceptado: 2 diciembre de 2019.

DOI.

Copyright: 2020 A.García, F.Garrido y J. Gallardo. This is an open access article under the CC BY-NC-SA 4.0 license (https://creativecommons.org/licenses/by-nc$\mathrm{sa} / 4.0 /)$. 
Para captar vibraciones en estructuras, existen muchos tipos de sensores, pero de alto costo; sin embargo, desde el año 2016 en la provincia de Chiriquí se ha implementado un método de captación de vibraciones en puentes de bajo costo aplicando el uso de la plataforma Arduino.

Para estudiar el comportamiento dinámico de una estructura, se puede utilizar el método matemático de análisis de Fourier, ya que permite identificar las frecuencias de vibración propias de dicho sistema, basándose en el hecho de que la función en el tiempo es periódica, y suponiendo un análisis dinámico de un sistema lineal [6].

En cada una de las tres investigaciones realizadas anteriormente en la provincia de Chiriquí por: Araúz \& Rodríguez [1], Patiño \& Reyes [7] y Serrano \& Zúñiga [8], las muestras de luces de acero fueron muy pequeñas en comparación con la cantidad de luces de concreto, por lo que no se pudieron obtener conclusiones definitivas sobre el comportamiento de puentes de acero. En este estudio se incluye una muestra considerable de luces de acero captada en las provincias de Veraguas y Coclé, en donde se observó un fenómeno de traslación de la frecuencia natural de aceleraciones verticales $(\mathrm{Az})$ de la luz más larga hacia sus luces adyacentes; por lo que se concluyó que la primera frecuencia que se observa en el espectro de las luces adyacentes es forzada, y la segunda frecuencia observada es la que pertenece naturalmente a dichas luces.

Se realizó un análisis de frecuencias de vibración vertical, utilizando los resultados obtenidos en investigaciones anteriores de luces de acero en la provincia de Chiriquí, en conjunto con la muestra de luces de acero de Veraguas y Coclé.

\section{Programa experimental}

Se monitoreó las aceleraciones verticales (Az) utilizando un equipo de medición ensamblado en una placa Arduino UNO, en un total de 31 luces con vigas de acero, en 11 puentes ubicados desde el corregimiento de San Martín de Porres, distrito de Las Palmas, provincia de Veraguas, hasta el corregimiento de Coclé, distrito de Penonomé, provincia de Coclé. El tramo en la Vía Interamericana, contemplado en el estudio, es de aproximadamente $162 \mathrm{~km}$, ver tabla 1 .
Tabla 1. Lista de puentes monitoreados en este estudio

\begin{tabular}{|c|c|c|}
\hline Puente & Luz & Código de Luz \\
\hline \multirow[t]{4}{*}{ Viguí (Vía David) } & L1 & L438281-906960 \\
\hline & $\mathrm{L} 2 *$ & L438264-906972 \\
\hline & L3 & L438242-906989 \\
\hline & L4 & L438220-907003 \\
\hline \multirow[t]{2}{*}{ Estero Salado (Vía Panamá) } & L1 & L543180-908757 \\
\hline & L2 & L543199-908766 \\
\hline \multirow[t]{3}{*}{ Pocrí (Vía David) } & L1* & L552260-914476 \\
\hline & $\mathrm{L} 2$ & L552254-914466 \\
\hline & $\mathrm{L}^{*} *$ & L552248-914453 \\
\hline \multirow[t]{3}{*}{ Chico (Vía Panamá) } & $\mathrm{L} 2 *$ & L553044-920100 \\
\hline & L3 & L553038-920117 \\
\hline & L4* & L553028-920147 \\
\hline \multirow[t]{5}{*}{ Chico (Vía David) } & L1 & L553000-920159 \\
\hline & $\mathrm{L} 2 *$ & L553002-920146 \\
\hline & L3 & L553007-920123 \\
\hline & $\mathrm{L}^{*} *$ & L553013-920100 \\
\hline & L5 & L553020-920078 \\
\hline \multirow[t]{3}{*}{ Churube (Vía David) } & L1 & L551776-927006 \\
\hline & $\mathrm{L} 2$ & L551777-926992 \\
\hline & L3 & L551779-926977 \\
\hline \multirow[t]{3}{*}{ El Caño (Vía David) } & $\mathrm{L} 1^{*}$ & L551599-928147 \\
\hline & $\mathrm{L} 2$ & L551601-928128 \\
\hline & $\mathrm{L} 3 *$ & L551606-928109 \\
\hline Salobre (Vía David) & $\mathrm{L}$ & L552089-929361 \\
\hline \multirow[t]{3}{*}{ Grande (Vía Panamá) } & L1 & L554795-931202 \\
\hline & L2 & L554808-931217 \\
\hline & L4 & L554884-931261 \\
\hline El Barrero (Vía Panamá) & $\mathrm{L}$ & L557633-933206 \\
\hline \multirow[t]{3}{*}{ Coclé (Vía Panamá) } & $\mathrm{L} 1 *$ & L562145-934705 \\
\hline & $\mathrm{L} 2$ & L562175-934718 \\
\hline & L3* & L562192-934729 \\
\hline
\end{tabular}

\subsection{Sistemas de mediciones de aceleraciones verticales} (Az)

En este estudio se realizaron mediciones con la plataforma Arduino Uno, utilizando el Sensor MPU6050. Para almacenar la información obtenida mediante el 
sensor, el sistema de mediciones cuenta con un adaptador de tarjeta microSD.

Se utilizó una fuente de energía portátil, que consta de seis baterías AA conectadas en serie, para facilitar el manejo y el transporte del dispositivo en campo.

En la figura 1 se presenta un esquema general de los componentes del sistema de mediciones, con la carcasa protectora de acrílico abierta para una mejor apreciación.

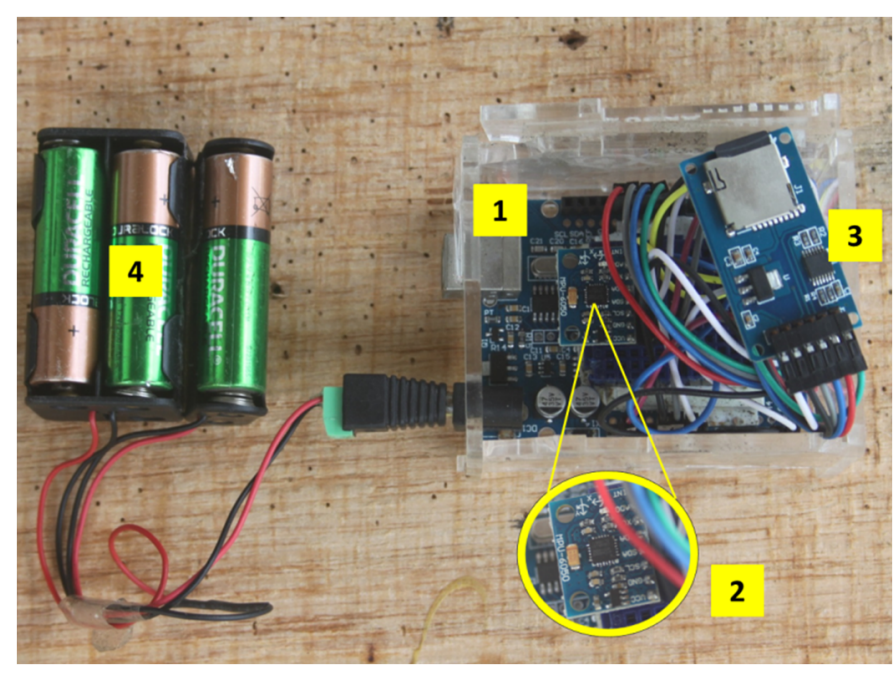

Figura 1. Sistema de mediciones con placa Arduino: 1) Placa Arduino. 2) Sensor MPU6050. 3) Adaptador MicroSD. 4) Fuente de energía.

\subsubsection{Almacenamiento de datos}

Debido a que este estudio requiere realizar largos viajes por carretera, es necesario tomar las medidas de forma rápida y eficiente para aprovechar al máximo cada jornada en campo, por lo que se necesitaba un dispositivo de mediciones inalámbrico. Para facilitar esta tarea era indispensable encontrar la manera de almacenar los datos captados en tiempo real y, posteriormente, realizar el respectivo análisis.

La unidad de almacenamiento debe ser pequeña y ligera, para que se ajuste al reducido espacio de la carcasa de acrílico del sistema de mediciones, por lo que se decidió instalar un adaptador de tarjeta de memoria micro SD a la placa microcontroladora Arduino UNO.

Los archivos se guardarán en formato CSV, los cuales pueden ser leídos con Microsoft Excel y no son de gran tamaño, por lo tanto, la capacidad de la tarjeta microSD no será de gran importancia, así que es recomendable comprar la más económica que se pueda conseguir.

\subsubsection{Fuente de energía}

Para seleccionar la fuente de alimentación se tomaron en cuenta las pruebas realizadas en tesis anteriores con diferentes tipos de baterías, en donde se concluyó que la mejor opción era usar 6 baterías AA recargables debido a su facilidad y factibilidad de recarga energética y ahorro económico [8].

Para armar esta fuente energética solo se necesita una porta baterías 6xAA con un conector de alimentación Jack macho de $5.5 \mathrm{~mm}$. En caso de no conseguir un porta baterías con todas estas especificaciones, se puede armar simplemente conectando varias porta baterías en serie y comprando por separado el cable de alimentación como se muestra en la figura 2.

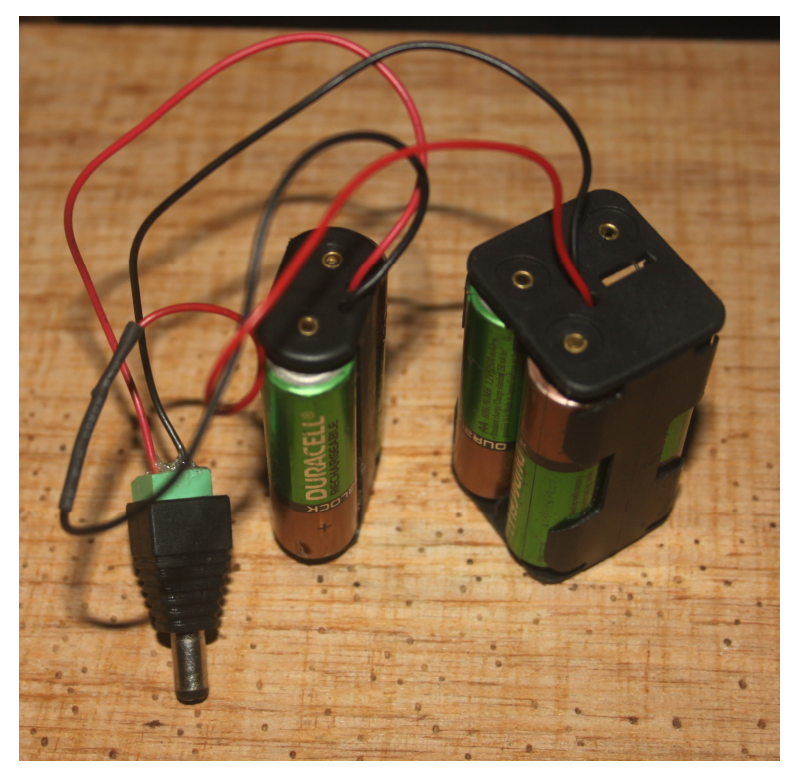

Figura 2. Fuente de alimentación de la placa Arduino UNO.

\subsection{Frecuencia de muestreo.}

Esta investigación se enfoca en obtener el primer modo de vibración de los puentes. Por lo tanto, se usará una frecuencia de muestreo que cumpla con el rango máximo, basándose en investigaciones anteriores realizadas en la provincia de Chiriquí, en donde el primer modo de vibración máxima tuvo un valor de $12.86 \mathrm{~Hz}$ en una de las luces del puente sobre el río Chiriquí Viejo [7].

A esta frecuencia mínima de muestreo con la que se puede trabajar para obtener las lecturas de una medición se le conoce como frecuencia Nyquist, la cual establece que, para poder captar la forma de una onda, la frecuencia de muestreo debe ser mayor al doble de la frecuencia que 
se desea medir [10]. Por lo tanto, la frecuencia de muestreo debe ser aproximadamente mayor a 26 lecturas por segundo.

Como resultado de distintas pruebas realizadas a nuestro sensor, se llegó a la conclusión de utilizar un tiempo entre cada lectura de 0.03 segundos para no tener fluctuaciones y poder captar todas las variables que se desean estudiar sin ningún problema. Esto equivale aproximadamente a una frecuencia de muestreo de 33.33 lecturas por segundo.

\subsection{Funcionamiento del sistema de mediciones.}

El dispositivo de esta investigación toma mediciones cada 30 milisegundos en un lapso de 10 minutos por cada luz del puente. Este se colocará a $0.4 \mathrm{~L}$, siendo L la longitud total de la luz.

La medición comienza en cuanto se conecta a la fuente de energía y termina en el momento en que se voltea simultáneamente, guardando los datos en la tarjeta microSD.

\subsection{Procesamiento de datos}

Los archivos guardados en la memoria microSD del dispositivo de mediciones contienen las lecturas obtenidas en cada medición en el orden en que fueron tomadas, guardadas en un formato .csv que se puede abrir en la computadora usando Excel®. Esta información se encuentra en su estado crudo, es decir, que necesita ser modificada antes de realizar cualquier análisis. Por lo tanto, este procesamiento de datos será dividido en dos etapas:

-Etapa I: Organización y edición de las lecturas obtenidas.

-Etapa II: Análisis de las mediciones mediante software.

\subsubsection{Etapa I: Organización y edición de las lecturas obtenidas.}

Es importante llevar un registro del orden en que se realizan las mediciones en cada puente, ya que lo primero que se debe hacer al adquirir los archivos almacenados por el dispositivo Arduino es renombrarlos para poder identificar cada archivo con la luz del puente al que pertenece. Para futuras investigaciones sería útil incluir sensores de señal GPS para que el dispositivo asigne el nombre al archivo automáticamente, basado en la ubicación del sensor al momento de la lectura.
Debido a interferencias que pueden ser captadas al momento de colocar y conectar el dispositivo en el lugar donde se desea realizar el monitoreo, es necesario eliminar cierta cantidad de lecturas iniciales en cada archivo. Por lo que, con la ayuda de Excel® se tomará una muestra de 20000 lecturas, lo que representa a 10 minutos de monitoreo por cada luz, omitiendo 30 segundos al inicio y al final

Es por esta razón, que cada medición se realizó en 11 minutos para evitar errores ocasionados por la manipulación del dispositivo al inicio y al final.

\subsubsection{Etapa II: Análisis de las mediciones mediante software}

Las medidas captadas en la primera etapa del procesamiento son aceleraciones en función del tiempo.

Para la detección de las frecuencias de los modos de vibración se realizó un procesamiento de datos que consiste en un análisis espectral aplicando la transformada rápida de Fourier a la data obtenida durante las mediciones con los sensores, lo que nos brindó los espectros de frecuencia de aceleraciones verticales en cada luz de puentes.

Para realizar la transformada rápida de Fourier se utilizó SPYDER (Scientific Python Development Environment). Últimamente, muchos investigadores y grupos de investigación, han iniciado una transición de MATLAB a PYTHON, debido a que el ecosistema científico de este último ha madurado rápidamente, presentando una alternativa muy atractiva de instalación gratuita y de código abierto [11]. Es por ello que en nuestra investigación se ha optado por utilizar el software de libre acceso llamado SPYDER escrito en lenguaje de programación PHYTON y con características similares a MATLAB.

La función de este código de programación consiste en aplicar la transformada rápida de Fourier a la muestra y mediante método de ventaneo, obtener gráficas de amplitud vs frecuencias, es decir, divide la muestra en múltiples secciones de igual tamaño y realiza un análisis independiente a cada parte. Esto permite diferenciar el valor de frecuencia de la estructura y omitir frecuencias pertenecientes a alguno de los distintos tipos de vehículos que transitan durante la prueba. 


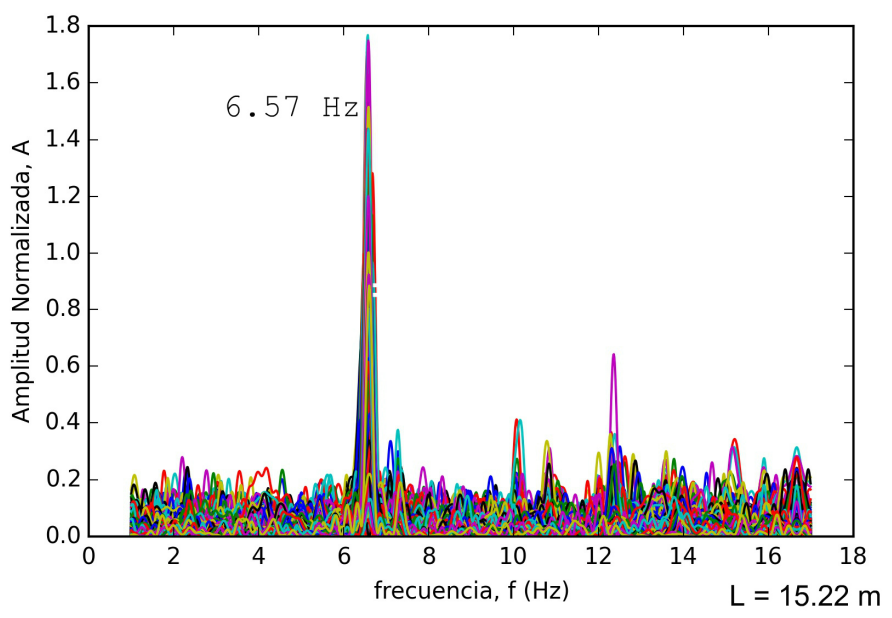

Figura 3. Ejemplo de gráfico obtenido con el software SPYDER del espectro Az en el puente sobre el río Churube, vía David, L2 de acero (L551777-926992).

\section{Análisis de Resultados}

Con el objetivo de identificar tendencias y robustecer la representatividad estadística del estudio, se amplió la muestra a un total de 56 luces incluyendo los resultados de tres estudios previos:

- Araúz \& Rodríguez [1]: iniciando desde el distrito de Boquerón; hasta el distrito de Tolé, en donde se tomaron 14 luces con vigas de acero.

Tabla 2. Lista de puentes de Araúz \& Rodríguez [1]

\begin{tabular}{|l|l|l|}
\hline \multicolumn{1}{|c|}{ Puente } & Luz & Código de Luz \\
\hline Chorcha & L1 & L357259-929175 \\
\cline { 2 - 3 } & L2 & L357248-929186 \\
\cline { 2 - 3 } & L3 & L357215-929186 \\
\hline La Pita & L & L361937-928739 \\
\hline Las Vueltas & L1 & L366375-928803 \\
\cline { 2 - 3 } & L2 & L366353-928814 \\
\hline San Juan & L1 & L393393-914210 \\
\cline { 2 - 3 } & L2 & L393382-914199 \\
\cline { 2 - 3 } & L3 & L393360-914177 \\
\cline { 2 - 3 } & L4 & L393349-914166 \\
\hline Tolé & L1 & L423328-913771 \\
\cline { 2 - 3 } & L2 & L423309-909338 \\
\cline { 2 - 3 } & L3 & L423287-909316 \\
\cline { 2 - 3 } & L4 & L423265-909305 \\
\hline
\end{tabular}

- Reyes \& Patiño [7]: Los puentes de este estudio se localizan específicamente desde el corregimiento de La Concepción, en el distrito de Bugaba, hasta la ciudad de Paso Canoas en el corregimiento de Progreso, en donde se tomaron cinco luces con vigas de acero.

Tabla 3. Lista de puentes Reyes \& Patiño [7]

\begin{tabular}{|l|c|c|}
\hline Puente & Luz & Código de Luz \\
\hline Mula & L & L322069-941128 \\
\hline \multirow{3}{*}{ Escarrea } & L1 & L317007-942708 \\
\cline { 2 - 3 } & L2 & L316986-942715 \\
\cline { 2 - 3 } & L3 & L316965-942723 \\
\hline Divala & L2 & L313840-943209 \\
\hline
\end{tabular}

- Serrano \& Zúñiga [8]: iniciando desde el distrito de Boquerón; hasta el distrito de David, en donde se tomaron seis luces con vigas de acero.

Tabla 4. Lista de puentes Serrano \& Zúñiga [8]

\begin{tabular}{|l|c|c|}
\hline \multicolumn{1}{|c|}{ Puente } & Luz & \multicolumn{1}{c|}{ Código de Luz } \\
\hline Chirigagua & L & L-330300E-936820N \\
\hline San Cristobal & L & L-340140E-931985N \\
\hline David & L1 & L-344780E-932535N \\
\cline { 2 - 3 } & L2 & L-344770E-932545N \\
\cline { 2 - 3 } & L3 & L-344755E-932560N \\
\cline { 2 - 3 } & L4 & L-344740E-932580N \\
\hline
\end{tabular}

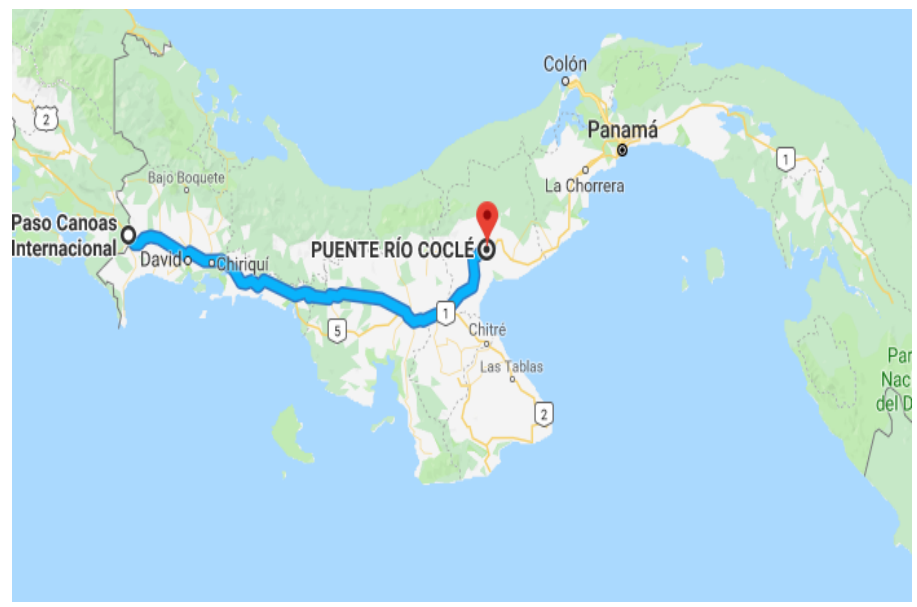

Figura 4. Tramo de la vía interamericana donde se han realizado estudio de puentes $(336 \mathrm{~km})$. 
Se tomó el primer modo de frecuencia de vibración, en cada luz de los estudios previos en provincia de Chiriquí, y se observó el fenómeno de traslado de frecuencia natural de la luz más grande hacia las luces adyacentes en los espectrogramas de frecuencia [9].

Se decidió tomar la segunda frecuencia del espectro, descartando la primera frecuencia de vibración forzada que se repite en las luces donde ocurrió el fenómeno antes mencionado; en las figuras 5 y 6 se pueden observar ejemplos de este fenómeno. En el caso del puente sobre el río Pocrí, la luz principal (de $15.22 \mathrm{~m}$ ) presenta una frecuencia de vibración natural de $6.39 \mathrm{~Hz}$; las luces adyacentes (de $11.75 \mathrm{~m}$ ) presentan una frecuencia natural de entre $8.48 \mathrm{~Hz}$ y $8.68 \mathrm{~Hz}$, sin embargo, en sus espectros se puede observar un máximo local de contenido para la frecuencia de $6.38 \mathrm{~Hz}$, que se puede considerar igual a la frecuencia de la luz central $(6.39 \mathrm{~Hz})$.

En el caso del puente sobre el Río Chico en Coclé, la luz central (de $27.4 \mathrm{~m}$ ) presenta una frecuencia de vibración natural de $3.04 \mathrm{~Hz}$; mientras que las luces adyacentes (de $22.1 \mathrm{~m}$ de luz) presentan un contenido de frecuencias importante en $3.04 \mathrm{~Hz}$, lo cual indica que las luces adyacentes están vibrando a la misma frecuencia que la luz central. Se considera que esta sincronía perfecta en la vibración puede deberse: (1) que las tres luces, siendo independientes, tienen la misma frecuencia natural (lo que es improbable, ya que la luz central es $19 \%$ mayor que las adyacentes), o, (2) dicha sincronización puede deberse a que la luz central induce a las luces adyacentes a vibrar, causando una vibración forzada, cuya frecuencia no depende de la longitud de las luces adyacentes, sino que depende de la fuente que la causa, en este caso: la luz central. En las luces adyacentes se observa adicionalmente un contenido de frecuencias importante para aproximadamente $4.54 \mathrm{~Hz}$; frecuencia que es muy cercana a la frecuencia esperada para esta luz (la frecuencia esperada es de $3.89 \mathrm{~Hz}$, según estimación presentada en la sección 4.1)

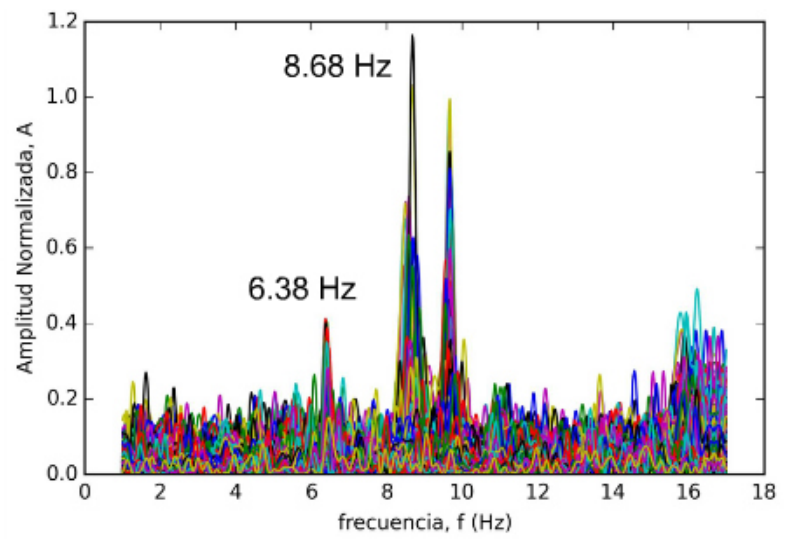

$\mathrm{L} 1=15.75 \mathrm{~m}$

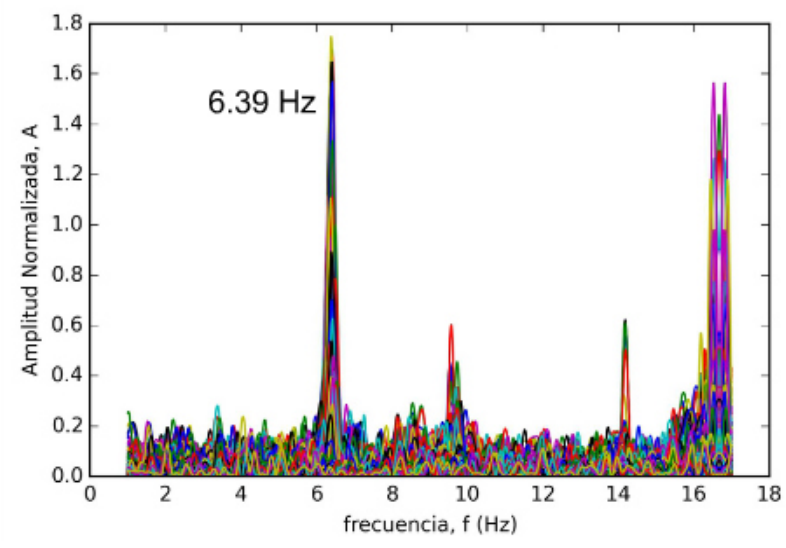

$\mathrm{L} 2=15.22 \mathrm{~m}$

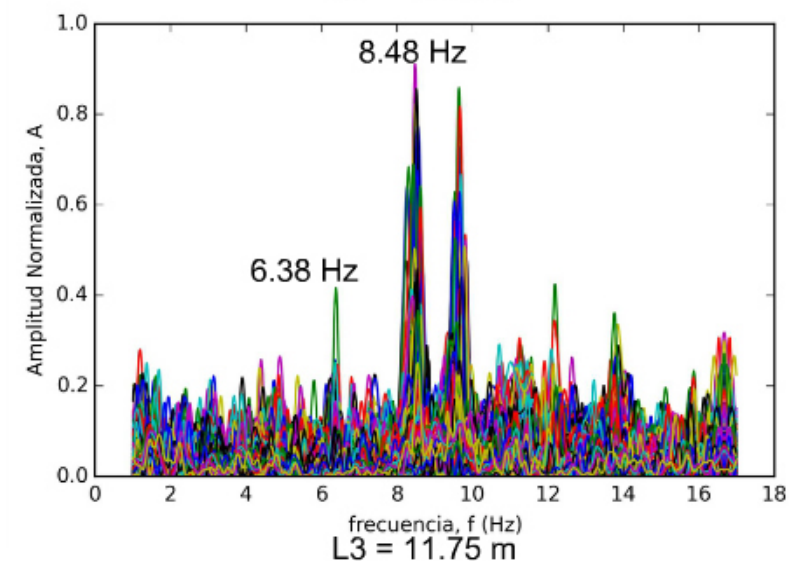

Figura 5. Ejemplo del fenómeno de traslación de frecuencias en el puente sobre el río Pocrí (vía David). L1 (L543199. 908766), L2 (L552254-914466) y L3 (L552248-914453). 

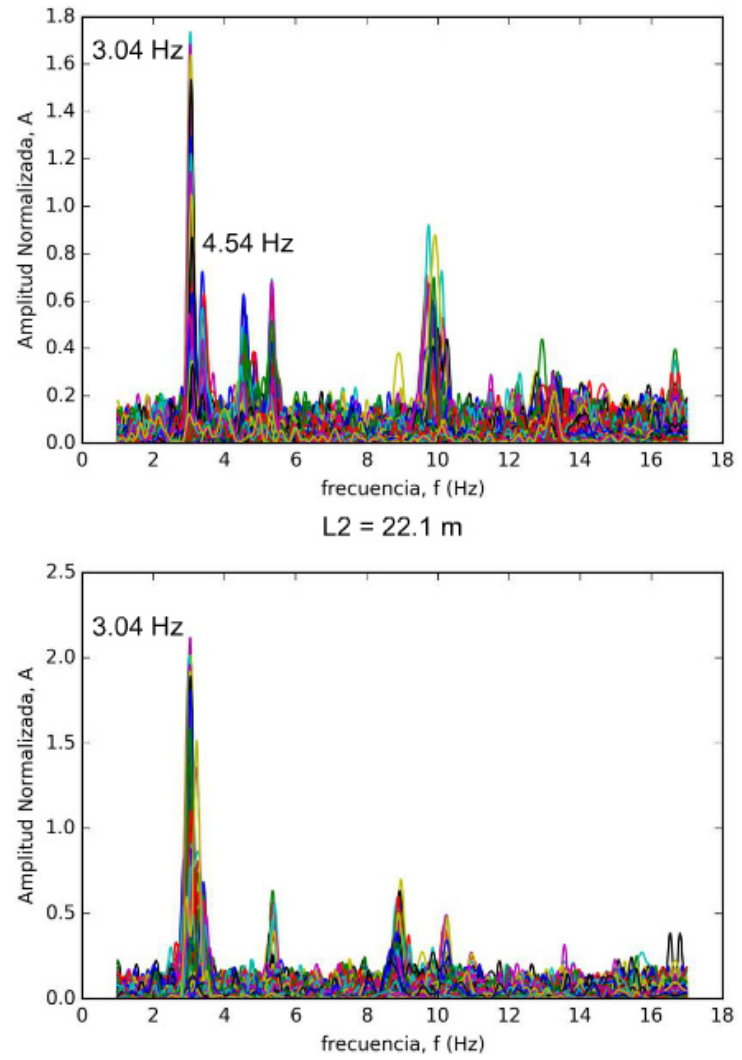

$\mathrm{L} 3=27.4 \mathrm{~m}$

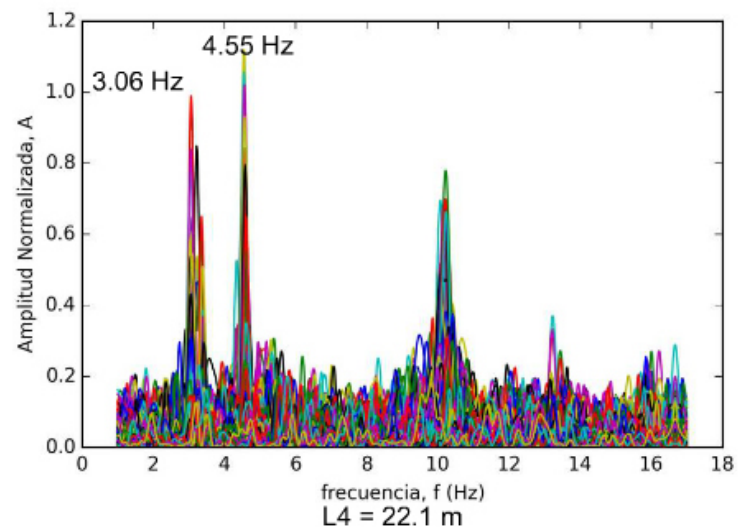

Figura 6. Ejemplo del fenómeno de traslación de frecuencias en el puente sobre el río Chico (provincia de Coclé) en las tres luces centrales (vía David). L2 (L553002-920146), L3 (L553007-920123) y L4 (L553013-920100).

A continuación, se presentan en las tablas: 5,6,7 y 8 los valores de la primera frecuencia natural de vibración para aceleraciones verticales Az obtenidos en las luces de los puentes con vigas de acero a lo largo de la vía interamericana estudiados hasta el momento.
Tabla 5. Valores de frecuencia para (Az) de la investigación Arauz \& Rodríguez [1]

\begin{tabular}{|c|c|c|c|c|}
\hline Puente & Luz & $\begin{array}{c}\text { Longitud, } \\
\text { L (m) }\end{array}$ & $\begin{array}{l}\text { Frecuencia, } \\
\text { f1 }(\mathrm{Hz})\end{array}$ & $\begin{array}{c}\text { Frecuencia } \\
\text { natural, f1 } \\
(\mathrm{Hz})\end{array}$ \\
\hline \multirow[t]{3}{*}{ Chorcha } & L1 & 23.3 & 3.51 & 3.51 \\
\hline & L2 & 27.4 & 3.27 & 3.27 \\
\hline & L3 & 23.3 & 3.51 & 3.51 \\
\hline La Pita & $\mathrm{L}$ & 27.8 & 3.41 & 3.41 \\
\hline \multirow{2}{*}{$\begin{array}{l}\text { Las } \\
\text { Vueltas }\end{array}$} & L1 & 10 & 9.13 & 9.13 \\
\hline & L2 & 12 & 9.13 & 9.13 \\
\hline \multirow[t]{4}{*}{ San Juan } & $\mathrm{L}^{*} *$ & 19.4 & 3.08 & 4.15 \\
\hline & $\mathrm{L} 2$ & 24.5 & 3.12 & 3.12 \\
\hline & L3 & 24.5 & 3.12 & 3.12 \\
\hline & L4* & 19.4 & 3.08 & 4.17 \\
\hline \multirow[t]{4}{*}{ Tolé } & L1 & 25 & 3.66 & 3.66 \\
\hline & L2 & 25 & 3.02 & 3.02 \\
\hline & L3* & 25 & 2.64 & 3.01 \\
\hline & L4 & 30 & 2.78 & 2.78 \\
\hline
\end{tabular}

Tabla 6. Valores de frecuencia para (Az) de la investigación Patiño \& Reyes [7]

\begin{tabular}{|l|c|c|c|c|}
\hline Puente & Luz & $\begin{array}{c}\text { Longitud, } \\
\text { L (m) }\end{array}$ & $\begin{array}{c}\text { Frecuencia, } \\
\text { f1 (Hz) }\end{array}$ & $\begin{array}{c}\text { Frecuencia } \\
\text { natural, f1 } \\
\text { (Hz) }\end{array}$ \\
\hline Mula & L & 30.92 & 2.73 & 2.73 \\
\hline Escarrea & L1 & 24.57 & 3.23 & 3.23 \\
\cline { 2 - 5 } & L2 & 30.46 & 2.88 & 2.88 \\
\cline { 2 - 5 } & L3 & 24.23 & 3.23 & 3.23 \\
\hline Divala & L2 & 30.8 & 2.73 & 2.73 \\
\hline
\end{tabular}

Tabla 7. Valores de frecuencia para (Az) de la investigación Serrano \& Zúñiga [8]

\begin{tabular}{|l|c|c|c|c|}
\hline \multicolumn{1}{|c|}{ Puente } & Luz & $\begin{array}{c}\text { Longitud, } \\
\text { L (m) }\end{array}$ & $\begin{array}{c}\text { Frecuencia, } \\
\mathbf{f 1}(\mathbf{H z})\end{array}$ & $\begin{array}{c}\text { Frecuencia } \\
\text { natural, } \\
\mathbf{f 1}(\mathbf{H z})\end{array}$ \\
\hline $\begin{array}{l}\text { Chirigagua } \\
\text { (aguas } \\
\text { arriba) }\end{array}$ & $\mathrm{L}$ & 29.2 & 3.75 & 3.75 \\
\hline $\begin{array}{l}\text { San } \\
\text { Cristobal } \\
\text { (aguas } \\
\text { arriba) }\end{array}$ & L & 15.5 & 8.27 & 8.27 \\
\hline & L1* & 20.2 & 3.84 & 4.5 \\
\hline
\end{tabular}




\begin{tabular}{|l|c|c|c|c|}
\hline \multirow{2}{*}{$\begin{array}{l}\text { David } \\
\text { (aguas } \\
\text { arriba) }\end{array}$} & L2 & 24.3 & 3.82 & 3.82 \\
\cline { 2 - 5 } & L3 & 24.4 & 3.83 & 3.83 \\
\cline { 2 - 5 } & L4* & 19.5 & 3.83 & 4.5 \\
\hline
\end{tabular}

Tabla 8. Valores de frecuencia para (Az) - este estudio

\begin{tabular}{|c|c|c|c|c|}
\hline Puente & Luz & $\begin{array}{c}\text { Longitud, } \\
\text { L (m) }\end{array}$ & $\begin{array}{c}\text { Frecuencia, } \\
\text { f1 }(\mathrm{Hz})\end{array}$ & $\begin{array}{l}\text { Frecuencia } \\
\text { natural, f1 } \\
(\mathrm{Hz})\end{array}$ \\
\hline \multirow{4}{*}{$\begin{array}{l}\text { Viguí } \\
\text { (Vía } \\
\text { David) }\end{array}$} & L1 & 21.6 & 4.8 & 4.8 \\
\hline & $\mathrm{L} 2 *$ & 24.5 & 2.82 & 4.2 \\
\hline & L3 & 30.4 & 2.83 & 2.83 \\
\hline & $\mathrm{L} 4$ & 24.5 & 4.23 & 4.23 \\
\hline \multirow{2}{*}{$\begin{array}{l}\text { Estero } \\
\text { Salado } \\
\text { (Vía } \\
\text { Panamá) }\end{array}$} & L1 & 21.45 & 3.73 & 3.73 \\
\hline & L2 & 21.45 & 3.82 & 3.82 \\
\hline \multirow{3}{*}{$\begin{array}{l}\text { Pocrí } \\
\text { (Vía } \\
\text { David) }\end{array}$} & L1* & 11.75 & 6.38 & 8.68 \\
\hline & L2 & 15.22 & 6.39 & 6.39 \\
\hline & L3* & 11.75 & 6.38 & 8.48 \\
\hline \multirow{3}{*}{$\begin{array}{l}\text { Chico } \\
\text { (Vía } \\
\text { Panamá) }\end{array}$} & $\mathrm{L} 2 *$ & 22.1 & 2.75 & 3.7 \\
\hline & L3 & 27.4 & 2.78 & 2.78 \\
\hline & L4* & 22.1 & 2.79 & 3.9 \\
\hline \multirow{5}{*}{$\begin{array}{l}\text { Chico } \\
\text { (Vía } \\
\text { David) }\end{array}$} & L1 & 15.5 & 6.09 & 6.09 \\
\hline & $\mathrm{L} 2 *$ & 22.1 & 3.04 & 4.54 \\
\hline & L3 & 27.4 & 3.04 & 3.04 \\
\hline & L4* & 22.1 & 3.06 & 4.55 \\
\hline & L5 & 15.5 & 6.04 & 6.04 \\
\hline \multirow{3}{*}{$\begin{array}{l}\text { Churube } \\
\text { (Vía } \\
\text { David) }\end{array}$} & L1 & 11.78 & 8.75 & 8.75 \\
\hline & L2 & 15.22 & 6.57 & 6.57 \\
\hline & L3 & 11.78 & 8.76 & 8.76 \\
\hline \multirow{3}{*}{$\begin{array}{l}\text { El Caño } \\
\text { (Vía } \\
\text { David) }\end{array}$} & $\mathrm{L} 1 *$ & 17.22 & 3.36 & 5.08 \\
\hline & L2 & 21.3 & 3.35 & 3.35 \\
\hline & L3* & 17.22 & 3.38 & 5.08 \\
\hline $\begin{array}{l}\text { Salobre } \\
\text { (Vía } \\
\text { David) }\end{array}$ & $\mathrm{L}$ & 30.5 & 2.34 & 2.34 \\
\hline \multirow{3}{*}{$\begin{array}{l}\text { Grande } \\
\text { (Vía } \\
\text { Panamá) }\end{array}$} & L1 & 24.7 & 3.04 & 3.04 \\
\hline & $\mathrm{L} 2$ & 24.7 & 3.2 & 3.2 \\
\hline & $\mathrm{L} 4$ & 24.7 & 3.15 & 3.15 \\
\hline $\begin{array}{l}\text { El } \\
\text { Barrero } \\
\text { (Vía } \\
\text { Panamá) }\end{array}$ & $\mathrm{L}$ & 21.7 & 5.22 & 5.22 \\
\hline
\end{tabular}

\begin{tabular}{|l|c|c|c|c|}
\hline $\begin{array}{l}\text { Coclé } \\
\text { (Vía }\end{array}$ & L1* & 24.5 & 2.55 & 3.8 \\
\cline { 2 - 5 } Panamá) & L2 & 30.4 & 2.56 & 2.56 \\
\cline { 2 - 5 } & L3* & 24.5 & 2.55 & 3.83 \\
\hline
\end{tabular}

\section{Análisis de resultados}

Anteriormente se determinó que existe una alta correlación entre el periodo de vibración de aceleraciones verticales Az y la longitud de las luces en los puentes de concreto [12]. Sin embargo, la cantidad de puentes con vigas de acero era muy escasa, por lo que no se pudo demostrar esta correlación.

No obstante, García \& Garrido presentaron a finales del 2018, una muestra mucho más grande de puentes de acero, por lo que sí se pudo observar y demostrar la correlación entre periodo y longitud de luz [8]. Por ello se decidió realizar un análisis con todos los resultados obtenidos hasta ahora en las provincias de Chiriquí, Veraguas y Coclé.

Mediante el análisis realizado por el software SPYDER, en el cual se obtuvieron gráficos de amplitud vs frecuencia, se puede observar la primera frecuencia perteneciente a cada luz de puente estudiada. Con esta primera frecuencia se puede calcular el periodo natural de vibración usando la siguiente ecuación:

$$
\mathrm{T}=\frac{1}{\mathrm{f}}
$$

Donde, la variable $\mathrm{T}$ representa al periodo en unidades de segundo y la variable $f$ es la frecuencia en unidades de $\mathrm{Hz}$.

\subsection{Ecuación de periodo vs longitud (Az)}

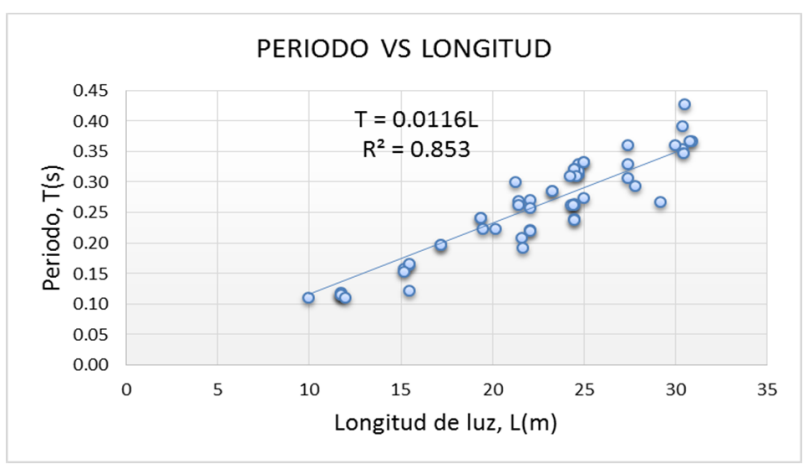

Figura 7. Periodo vs longitud de luz $(n=56)$.

En la figura 7 se muestra la correlación que, entre el periodo de vibración y la longitud de luz para puentes con vigas de acero, de esta manera podemos estimar que 
la ecuación para el periodo esperado en este tipo de puentes es la siguiente:

$$
\mathrm{T}=(1 \mathrm{~s})\left(\frac{L}{86 m}\right)
$$

Se observa una alta correlación entre el periodo y la longitud de las 56 luces de puentes con vigas de acero estudiados $\left(\mathrm{R}^{2}=0.853\right)$.

\subsection{Ecuación de tendencia de límite superior}

Para calcular la ecuación de límite superior de tendencia, se realizará la misma metodología que se utilizó para puentes con vigas de concreto [1], utilizando ahora la ecuación de tendencia obtenida en esta investigación para puentes con vigas de acero, ver tabla 9.

Tabla 9. Valores estadísticos

\begin{tabular}{|l|l|}
\hline \multicolumn{1}{|c|}{ Función } & Valor \\
\hline Desviación estándar & 0.122 \\
\hline Promedio & 0.979 \\
\hline $\begin{array}{l}\text { Desviación estándar } \\
\text { relativa }\end{array}$ & 0.124 \\
\hline Factor de límite superior & 0.0128 \\
\hline
\end{tabular}

Ecuación de límite superior para puentes con vigas continuas de concreto:

$$
\mathrm{T}=0.0128 \mathrm{~L}
$$

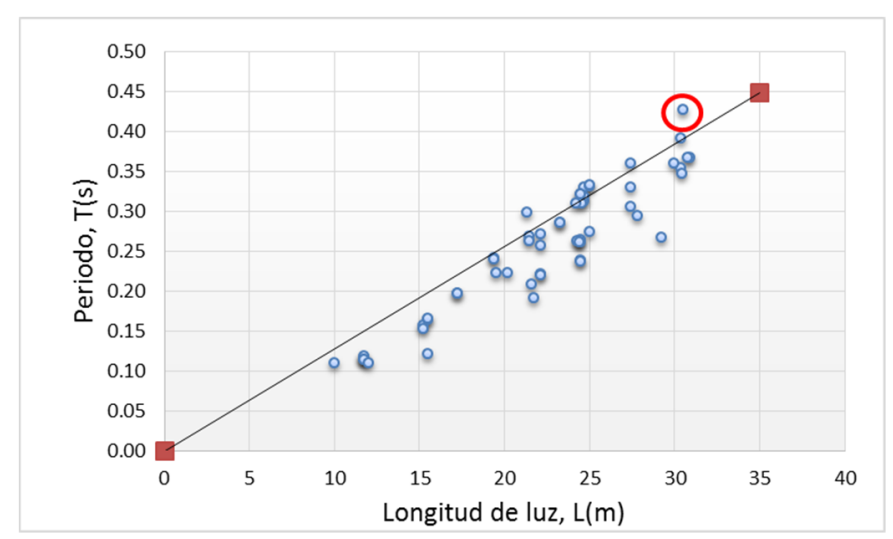

Figura 8. Línea de tendencia de límite superior.

En la figura 4 se observa que la única luz del puente sobre el río Salobre (Vía David), la luz número tres del puente sobre el río Chico (Vía Panamá), la luz número uno del puente sobre el río Grande (Vía Panamá), la luz número dos del puente sobre el río El Caño (Vía David) y la luz número dos del puente sobre el rio Tolé presentan valores por encima del límite superior.

\section{Conclusiones}

De acuerdo con los resultados obtenidos en este estudio se puede llegar a las siguientes conclusiones:

1. Se encontró una alta correlación en el gráfico de periodo vs longitud de las 56 luces de puentes con vigas de acero estudiados.

2. Se determinó una ecuación del periodo de las luces de acero en función de su longitud (ecuación 2), Las estimaciones realizadas con esta ecuación presentaron un coeficiente de variación (de las estimaciones con respecto a las mediciones) del $12 \%$.

3. Mediante análisis estadístico se generó la ecuación (3), mediante la cual se define un límite superior propuesto para la frecuencia de vibración principal de los puentes de viga de acero, por sobre el cual se estima que el puente presenta una frecuencia de vibración atípica.

4. De las 56 luces de puentes con vigas de acero estudiadas, cinco luces están por encima de la línea de tendencia de límite superior: la única luz del puente sobre el río Salobre (Vía David), la luz número tres del puente sobre el río Chico (Vía Panamá), la luz número uno del puente sobre el río Grande (Vía Panamá), la luz número dos del puente sobre el río El Caño (Vía David) y la luz número dos del puente sobre el río Tolé.

\section{Recomendaciones}

Basado en los resultados de este estudio se puede recomendar:

1. Realizar este tipo de mediciones a una mayor cantidad de puentes con vigas de acero de gran longitud y con cerchas, para tener más cantidad de data de este tipo de puentes.

2. Llevar a cabo una inspección en el puente sobre el río Salobre (Vía David), ya que ha presentado un comportamiento atípico en base a este análisis $\mathrm{y}$ en los diferentes tipos de mediciones realizadas en la última investigación.

3. Hacer mediciones en los puentes con vigas de acero faltantes en la carretera interamericana 
para de esta manera tener una base de datos completa.

4. En futuras investigaciones realizar modelos matemáticos que sirvan para verificar la naturaleza del fenómeno de traslado de frecuencia, el cual no se puede explicar con modelos sencillos que consideren que los extremos de las vigas no continuas están unidos a apoyos rígidos.

\section{Referencias}

[1] S. Rodríguez O. Araúz, "Identificación estructural de puentes", I+D Tecnológico, vol. 13, no. 1, p 83-90, junio, 2017.

[2] W. Rücker, F. Hille and R. Rohrmann, "Guideline for the assessment of existing structures," Federal Institute of Materials Research and Testing, Germany, 2006.

[3] The manual for bridge evaluation. Washington, D.C.: American Association of State Highway and Transportation Officials, 2011, pp. 8.1-8.16.

[4] F. Navarro and D. Ureña, "Mediciones y análisis de vibraciones en el puente del Virilla", Investiga TEC, vol. 22, p. 16, 2015.

[5] J. Cortés, F. Medina and J. Chaves, "Del análisis de fourier a las wavelets análisis de fourier.", Scientia et technica, vol. 13, no. 34, 2007.

[6] Secretaría de comunicaciones y transportes, "Evaluación de puentes mediante el análisis de vibraciones", Instituto Mexicano del Transporte, Sanfandila, Qro, 1999.

[7] E. Patiño and E. Reyes, "Estudios estructurales de puentes basado en vibraciones en la región occidental de Chiriquí", Tesis Licenciatura en Ingeniería Civil, Universidad Tecnológica de Panamá, 2017.

[8] N. Serrano, B. Zuñiga, and J. Gallardo "Método fotogramétrico pragmático para medir desplazamientos en puentes". I+ D Tecnológico, vol. 14, no. 1, p 29-40, junio, 2018.

[9] Á. García and F. Garrido, "Identificación estructural de puentes entre Veraguas y Coclé por medio de su vibración y desplazamiento", Tesis de Licenciatura en Ingeniería Civil, Universidad Tecnológica de Panamá, 2018.

[10]J. Errington, "John Errington's Data Conversion Website", Skillbank, 2011. [Online]. Available: http://www.skillbank.co.uk/SignalConversion/rate.htm.

[11] "Python vs Matlab", Pyzo. [Online]. Available: https://pyzo.org/python_vs_matlab.html. 